Modern Physics Letters B, Vol. 21, No. 6 (2007) 303-319

(C) World Scientific Publishing Company

\title{
MAGNETIC PROPERTIES OF ORGANIC COATED GOLD SURFACES
}

\author{
J. DE LA VENTA, E. FERNANDEZ PINEL, M. A. GARCIA, \\ P. CRESPO and A. HERNANDO* \\ Instituto de Magnetismo Aplicado and Depto. Física de Materiales, \\ UCM-ADIF-CSIC P. O. Box 155, 28230 Las Rozas, Madrid, Spain \\ *antonio.hernando@adif.es \\ O. RODRIGUEZ DE LA FUENTE \\ Depto. Física de Materiales, Universidad Complutense, 28040, Madrid, Spain \\ C. DE JULIÁN FERNÁNDEZ \\ Dipartamento di Física "G. Gallilei" Università di Padova, \\ Via Marzolo 8, 35131 Padova, Italy \\ A. FERNÁNDEZ \\ Instituto de Ciencia de Materiales de Sevilla CSIC-UNSE, \\ and Departamento de Quimica Inorgánica, \\ Universidad de Sevilla, Spain \\ Américo Vespucio s/n, 41092 Sevilla, Spain

\section{S. PENADÉS} \\ Grupo Carbohidratos, Lab. of Glyconanotechnology IIQ-CSIC, \\ Américo Vespucio s/n, 41092 Sevilla, Spain
}

Received 10 February 2007

\begin{abstract}
We review here our recent results of experimental observation of room temperature magnetism in gold nanoparticles (NPs) and thin films. Capping gold surfaces with certain organic molecules leads to the appearance of magnetism at room temperature. The surface bonds between the organic molecules and $\mathrm{Au}$ atoms give rise to magnetic moments. These magnetic moments are blocked along the bond direction showing huge anisotropy. In the case of atomically flat surfaces, the magnetic moments are giants. An explanation of this orbital ferromagnetism is given. These results point out the possibility to observe magnetism at nanoscale in materials without typical magnetic atoms (transition metals and rare earths), and are of fundamental value to understand the magnetic properties of surfaces.
\end{abstract}

Keywords: Nanomagnetism; surface magnetism; nanoparticles. 


\section{Introduction}

The presence of hysteresis in the magnetic field dependence of the magnetization of ferro and ferrimagnetic materials is based on three elements: ${ }^{1}$ the existence of magnetic moments (held by electrons), the exchange interactions (that tend to keep these moments parallel) and the magnetic anisotropy (that gives preferential spatial orientation of the magnetic moments). The combination of these three elements gives rise to the so-called "ferromagnetic behavior": stable orientation of magnetic moments in certain spatial directions even in absence of magnetic fields, yielding to a macroscopic magnetization not zero. The main limitation to observe ferromagnetism in materials is due to the exchange interactions: many elements show magnetic moments and anisotropy is always present in crystal structures but only few elements of the periodic table exhibit exchange interactions strong enough to promote magnetic order. Hence, the magnetism is limited to few transition metals, rare earths and their alloys.

While these ideas are valid for the bulk, things are different at the nanoscale, where the properties of the materials are modified because of both size and surface effects, and new physics appears. One of the most striking features of nanostructures is that these systems present a large fraction of surface atoms. Figure 1 shows the fraction of surface atoms with respect to the total atoms in a spherical particle. As the size of particle decreases, there is a huge increase in this fraction. Therefore for particles of few nanometers of size (that is, NPs), surface effects become very important.

These surface atoms are different to those of volume and therefore, when they represent a non-negligible fraction of the total, the physical properties of the material may be modified. Moreover, the properties of the surface atoms can be also customized by bonds with other molecular species that alter their electronic structure and consequently all the physical properties depending on the electronic states. Actually, some surprising results related to the magnetic properties of nano-

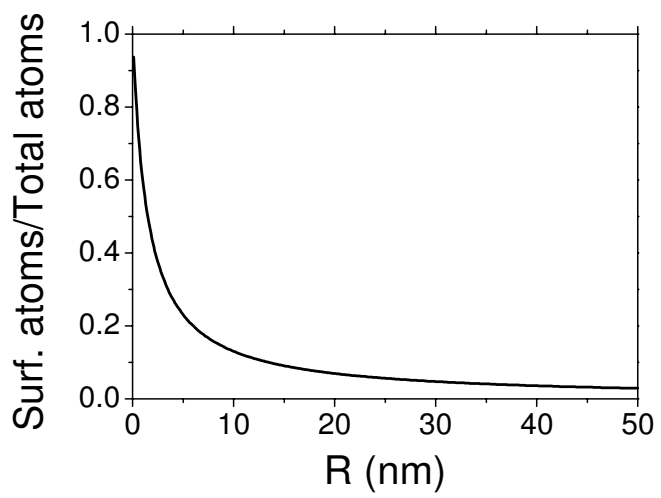

Fig. 1. Ratio of surface atoms with respect to the total atoms as a function of the particle's radius $R$, considering spherical sized NPs with an interatomic distance $0.3 \mathrm{~nm}$. 
structures and surfaces have been already observed, for instance in proton irradiated graphite $^{2}$ and dielectric oxides. ${ }^{3}$ An outstanding example of these surface effects is the appearance of huge magnetic anisotropy that blocks the magnetic moments of single Co atoms deposited onto Pt surfaces reported by Gambardella et al. ${ }^{4}$ Here, we illustrate how Au atoms, in surfaces and as clusters, which are normally diamagnetic, exhibit magnetic moments when they are interacting chemically with organic molecules.

\section{Au Nanoparticles}

A very striking result is the recent observation of magnetism ${ }^{5,6}$ in Au NPs capped with dodecanethiol, despite the diamagnetic character of bulk $\mathrm{Au}$ and thiols separately.

Calculations of the electronic structure of gold indicate that the $d$-band lies well below the Fermi level, where the density of states, $n\left(E_{\mathrm{F}}\right)=0.29 \mathrm{eV}^{-1}$ atom $^{-1}{ }^{7}$ is low enough to promote noticeable Pauli paramagnetism. Consequently, the weak $5 d$-band paramagnetism is overcome by the combination of Landau and core diamagnetism. As a result, bulk gold is diamagnetic with a susceptibility $\chi=-1.4 \times 10^{-7} \mathrm{emu} / \mathrm{gOe},{ }^{8}$ and is very far of presenting a ferromagnetic-like behavior.

However, as it is shown in Fig. 2(a), thiol-capped gold NPs of $1.4 \mathrm{~nm}$ size show permanent magnetism and hysteresis up to room temperature. ${ }^{5}$ The coercive field ranges from $85 \mathrm{Oe}$ at $5 \mathrm{~K}$ to $10 \mathrm{Oe}$ at $300 \mathrm{~K}$, as the inset shows. On the other side, Au NPs with similar size but stabilized by means of a surfactant — i.e. weak interaction between protective molecules and Au surface atoms - are diamagnetic as bulk Au samples are, see Fig. 2(b).

Zhang et al. showed that the capping molecule induces changes in the electronic configuration of the NPs. ${ }^{9,10}$ They found that Au NPs gain $d$-electron when capped

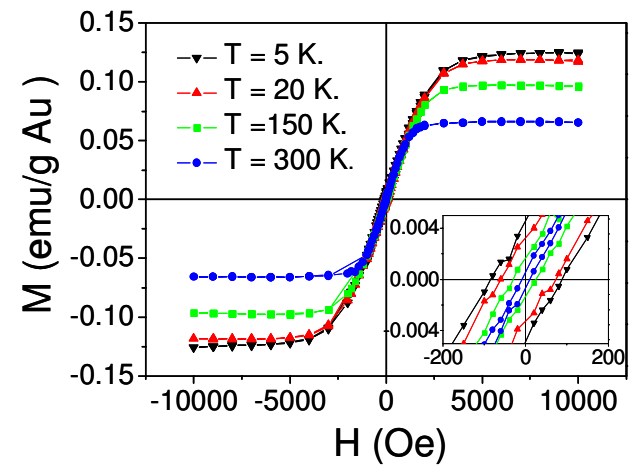

(a)

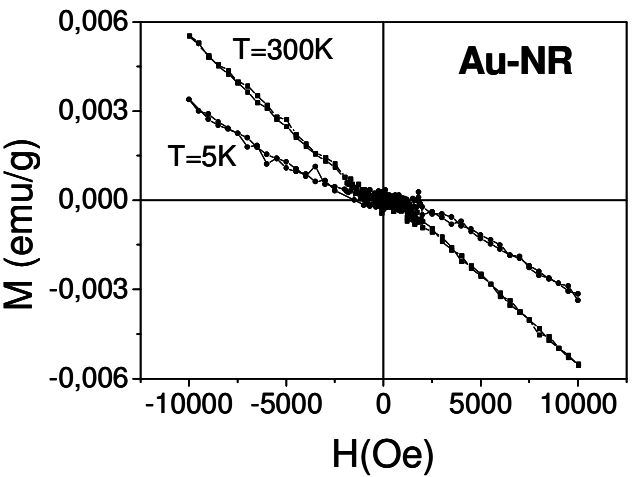

(b)

Fig. 2. Magnetization curves for NPs capped with (a) dodecanethiol and (b) tetraalkylammonium salts. Adapted from Ref. 5. 
with weakly interacting dendrimers (amine and alcohol groups). However, Au NPs lose $d$-electrons when capped with strongly interacting thiols. We confirmed, by XANES measurements that the spectra showed that for thiol-capped Au NPs there is a charge transfer from the $\mathrm{Au}$ to the $\mathrm{S},{ }^{5}$ so the $\mathrm{Au} 5 d$-band is incomplete and could stand a magnetic moment. Nevertheless, the charge transfer is absent for the diamagnetic samples capped with the surfactant. ${ }^{5}$ The surprising ferromagneticlike behavior is consequently associated with $5 d$-localized holes generated through $\mathrm{Au}-\mathrm{S}$ bonds.

It is consequently a surface effect: the magnetism arises at the NPs surface atoms. Therefore, the value of the magnetic moment per particle will depend on the NP size, which can be controlled through the preparation method. The Au NPs with dodecanethiol as the capping molecule were synthesized following the method described by Brust and co-workers, ${ }^{11}$ using two-phase reduction (watertoluene). Among the multiple preparation techniques of metallic NPs, the chemical synthesis is the most developed, is faster and cheaper than other physical techniques and allows an accurate control over the size and shape of the NPs. The chemical synthesis consists in the reduction of a metal salt precursor, in a liquid phase, in presence of "protective" species that, due to the formation of covalent links or by electrostatic interactions, isolate the metal cluster preventing its growth. A gold metallic salt, tetrachloroauric (III) acid $\left(\mathrm{HAuCl}_{4}\right)$, in aqueous solution is first transferred to an organic medium, toluene, with the help of a phase transfer reagent, tetraoctylammonium bromide $\left[\mathrm{CH}_{3}\left(\mathrm{CH}_{2}\right)_{7}\right]_{4} \mathrm{~N}(\mathrm{Br})$. In this organic medium, the metallic salt is reduced in presence of a capping molecule, in our case dodecanethiol $\left(\mathrm{CH}_{3}\left(\mathrm{CH}_{2}\right)_{11} \mathrm{SH}\right)$. The particle size is controlled mainly by the molar ratio of the metallic salt with respect to the thiol $\left(\mathrm{AuCl}_{4}^{-}: \mathrm{RSH}\right)$ and also by the temperature of the reaction and the rate of reductant addition. ${ }^{12}$ The increase in the amount of thiol produces a decrease in size of the NPs, because the metallic clusters are rapidly capped due to the excess of thiol. In addition, the faster the reductant addition, the narrower the NP size distribution. Consequently, we can achieve a good control over the NP size by controlling these parameters.

Figure 3 shows the diffractograms for Au NPs prepared following the method described above with different Au:S ratio. The broadening of the maxima confirms that there is a decrease in the size when the amount of thiol is increased.

This decrease in size is confirmed by atomic force microscopy (AFM). The characterization of NPs with an AFM is not straightforward because we are in the limit of resolution of this technique. Consequently, the conditions must be optimum and are well described in the literature. ${ }^{13}$ Actually, when observing small NPs onto a substrate by AFM, only the NPs height is well measured while the width is fairly distorted due to the convolution with the tip shape. ${ }^{13}$ Figure 4 shows an AFM image of NPs with approximately $5 \mathrm{~nm}$ of size.

The optical properties of the NPs have also been studied. The surface plasmon resonance (SPR) is the most remarkable optical property of metallic NPs. ${ }^{14,15}$ 


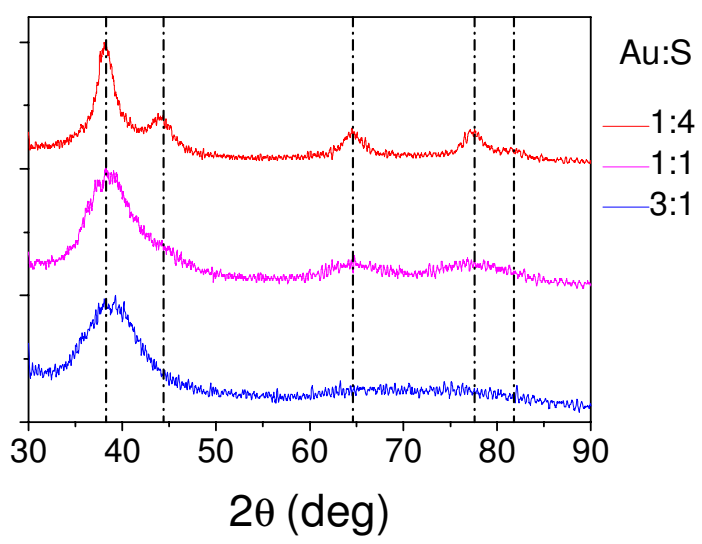

Fig. 3. X-ray diffractograms for three samples of $\mathrm{Au}$ NPs with different $\mathrm{Au}: \mathrm{S}\left(\mathrm{HAuCl}_{4}\right.$ :thiol) ratio. Dashed lines indicate the maxima position for the fcc bulk gold.

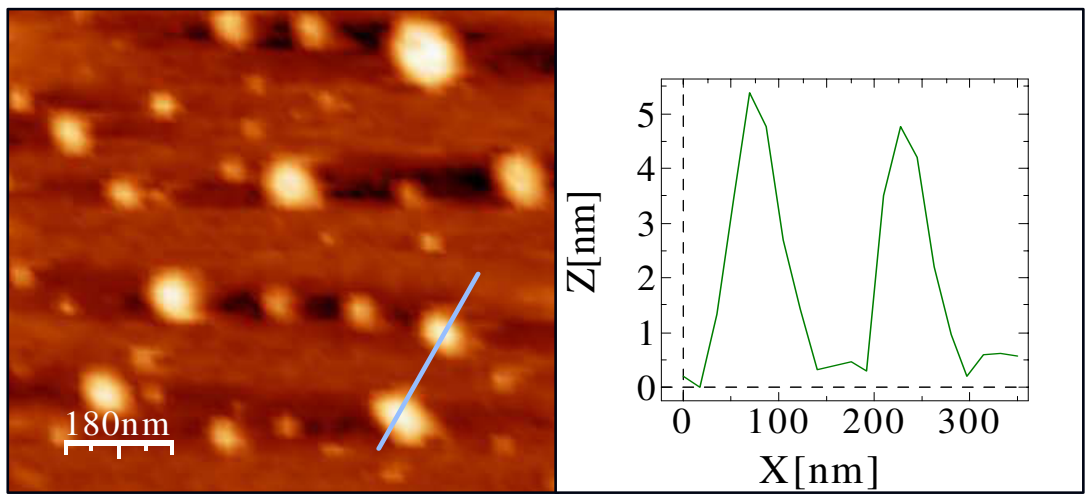

Fig. 4. AFM image from thiol-capped Au NPs and height profile along the indicated line.

SPR consists in a collective oscillation of the conduction electrons inside the NP. When an external electromagnetic field is applied, an excess of charge arises at the NP surface. This excess of charge acts as a restoring force, while the electron movement is damped mainly because of the electron interactions with atomic cores and NP surface. Hence, the system acts as a damped oscillator which presents a resonance frequency that for most of the transition metals lies on the UV-Vis part of the spectrum. ${ }^{16}$ The NPs exhibit an absorption band in this region of the optical absorption spectrum. According to the Mie theory, ${ }^{14,17}$ as the damping constant depends strongly on the particle size, the shape of the SPR band also does.

In Fig. 5, we show the calculated absorption spectrum for gold NPs of different sizes, following the Mie theory. The absorption band is centered in $2.4 \mathrm{eV}$. For small particles there is a broadening in the SPR. The full width half maximum (FWHM), 


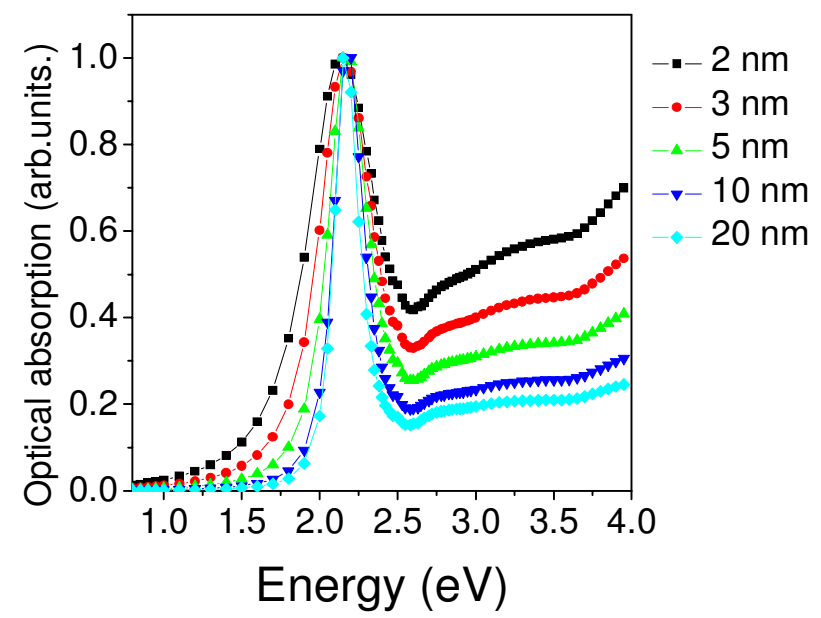

Fig. 5. Calculated absorption band following the Mie theory for Au NPs with different sizes. Adapted from Ref. 18.

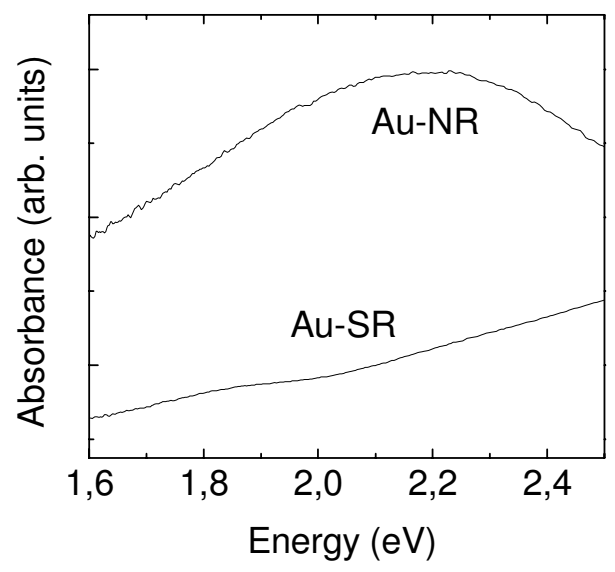

Fig. 6. Optical absorption spectra of Au NPs of $1.5 \mathrm{~nm}$ capped with tetraalkylammonium salts $(\mathrm{Au}-\mathrm{NR})$ and with dodecanethiol (Au-SR). Adapted from Ref. 5.

$\Gamma$ of the SPR band is related to the particle size through the equation:

$$
\Gamma=\Gamma_{0}+\frac{a}{R},
$$

$\Gamma_{0}$ and $a$ being constants that depends on the particular metal and $R$ the particle's radius.

However, we found that for Au NPs capped with different organic molecules, the width of the SPR is not determined only by the particle volume but also by the capping molecule. ${ }^{18}$ In $1.5 \mathrm{~nm}$ size NPs (Fig. 6), the thiol-capping yields the absence of SPR absorption band while when they are capped with other molecules (tetraalkylammonium in this case) the absorption band is still present. 


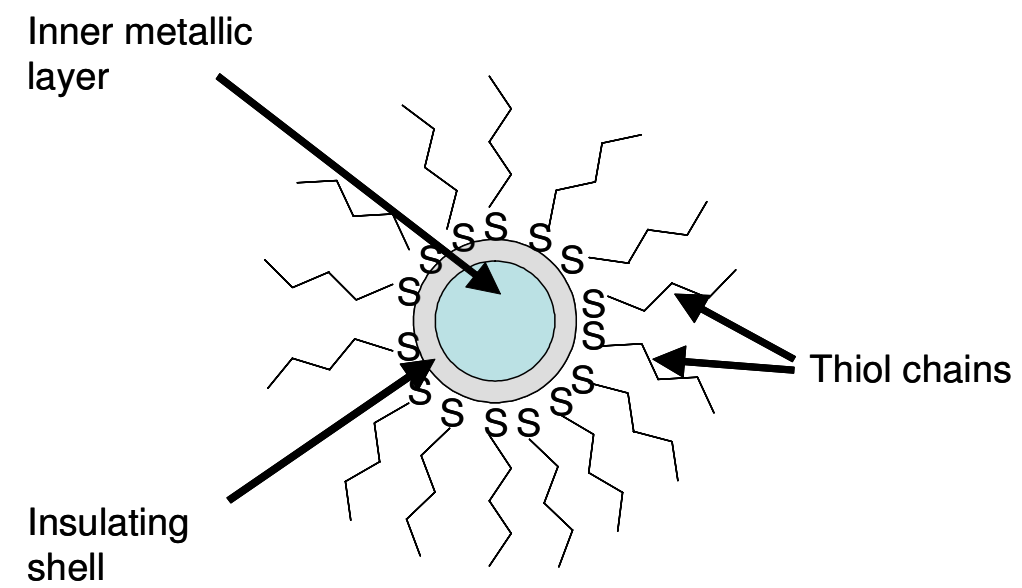

Fig. 7. Scheme of a thiol-capped Au NP with an external insulating shell where electron movement is damped by interaction with thiols, and an inner metallic core. Adapted from Ref. 18.

The tetraalkylammonium interact very weakly with the NPs and the capping agent does not modify their electronic structure. However, dodecanethiol is a strong interacting agent. For NPs capped with strongly interacting agents, the capping molecule does not merely passivate the metallic NPs but also modifies their electronic structure. These changes in the electronic density at the surface can induce an extra damping, which is called chemical interface damping. ${ }^{14,15}$ This produces a shell where electrons cannot oscillate because of charge localization in the $\mathrm{Au}-\mathrm{S}$ bond. Figure 7 shows a scheme of a thiol-capped Au NP with an external shell where electron movement is damped by interaction with thiols, and an inner core. If the NP is small enough, all the electrons are blocked, since the external shell insulates all the NP and this causes complete extinction of the SPR absorption band.

Recalling the surprising ferromagnetic-like behavior observed in thiol-capped $\mathrm{Au}$ NPs, we have also observed that it is strongly dependent on the particle size. For NPs with $2.5 \mathrm{~nm}$ of size (Fig. 8(a)), the magnetic measurements show a ferromagnetic-like behavior, with hysteresis up to room temperature. However, NPs of $6 \mathrm{~nm}$ (Fig. 8(b)) presents a diamagnetic behavior for high magnetic fields and a ferromagnetic-like in the central part of the magnetization curve.

To explain this behavior we suggest that the NPs have two different regions as is described in Fig. 7: the inner metallic core, which presents a diamagnetic behavior similar to bulk gold, and the outer shell (with the $\mathrm{Au}-\mathrm{S}$ bond), which has a ferromagnetic-like behavior.

The addition of these two effects gives rise to different magnetic behaviors as shown before. In NPs of $6 \mathrm{~nm}$, the diamagnetic contribution is dominant under a high magnetic field. This is because the strongest contribution is due to the inner core since the ratio of surface atoms with respect to the volume atoms is very small 


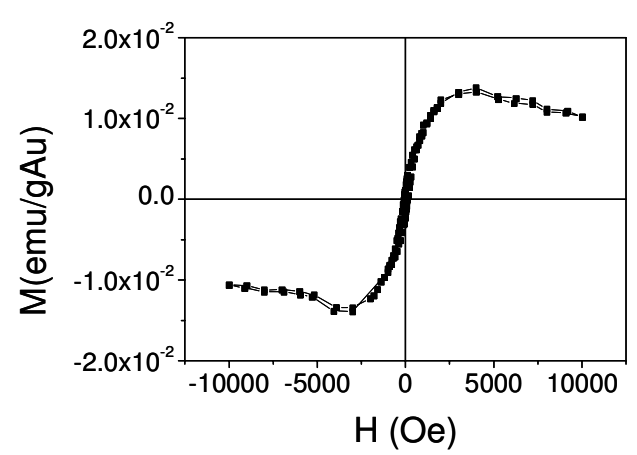

(a)

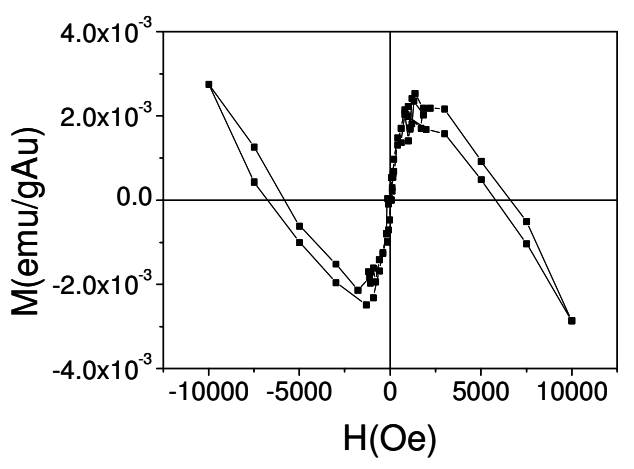

(b)

Fig. 8. Magnetization curves at $300 \mathrm{~K}$ for thiol-capped Au NPs with (a) $2.5 \mathrm{~nm}$ and (b) $6 \mathrm{~nm}$.

(see Fig. 1). However, in small particles, surface atoms become more important. Therefore, the $\mathrm{Au}-\mathrm{S}$ shell has a strong contribution, against the metallic core, and the ferromagnetic contribution is the most important.

According to these experiments, to explain the ferromagnetic-like behavior we suggest that the thiol chain joins to the gold through the gold-sulfur bond. This produces a charge transfer, as demonstrated by the XANES spectra. ${ }^{5}$ This charge is localized, as it was shown with the UV-Vis measurements, which gives rise to a magnetic moment. There is also an anisotropy axis in the direction of the thiol chain that in contribution with the spin-orbit coupling produces the blocking of the magnetic moments. Therefore, the charge localization and the anisotropy are responsible for the ferromagnetic-like behavior.

\section{Au Thin Films}

Thin films are also structures with a large fraction of surface atoms, in which the modification of surface by bonding with certain species could promote important modifications of their physical properties. Moreover, they supply a unique opportunity to study the anisotropy of surfaces, since whereas in the case of NPs the surface bonds are oriented in all possible directions, as it is illustrated in Fig. 9, there is a single orientation for thin films surfaces.

As for the case of NPs, also for thin films, some published experiments can be considered as hints that capping the surface with certain organic molecules yields to the appearance of ferromagnetic-like behavior at room temperature. In their work about the magnetism of Au surfaces capped with organic molecules, ${ }^{19}$ Carmeli et al. found that gold surfaces coated with a polyalanine monolayer chemisorbed on the gold film, present a ferromagnetic-like component at room temperature (Fig. 10). They found giant magnetic moments $\left(\sim 50 \mu_{B}\right.$ per surface atom). The substrate presents a diamagnetic behavior. This magnetic signal decays with time, due to the

degradation of the organic layer. 

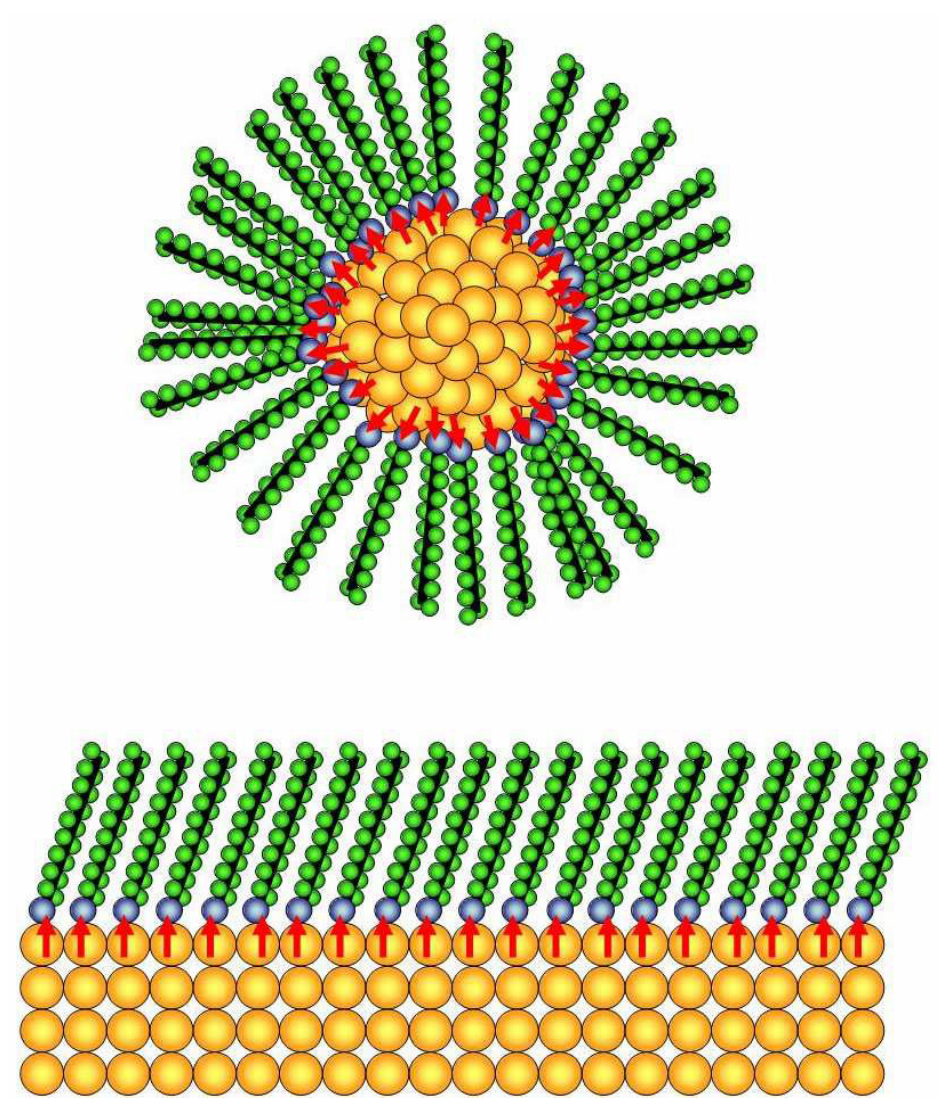

Fig. 9. Scheme of the magnetic moments arising at surfaces capped with organic molecules. The magnetic moments are fixed in the bond direction that for NPs are uniformly distributed, while for films there is a unique orientation perpendicular to the surface.

We prepared $\mathrm{Au}$ thin films capped with different organic molecules following the stripped gold template method. ${ }^{20,21}$ Briefly, a gold layer was deposited onto a cleaved mica substrate. The mica piece was next glued onto glass substrate. Finally, the mica sheet was removed using a blade. This method allows obtaining in a simple way an atomically flat gold (111) surface. Immediately after, self assembled monolayers (SAMs) were obtained by immersing the Au thin films in a solution containing the organic molecules, in this case a Lewis Neoglyconjugate. ${ }^{22}$ This molecule has at the end a thiol group that joins to the organic surface, via a $\mathrm{Au}-\mathrm{S}$ bond. After the absorption, the surface was rinsed with ultrapure water and dried under $\mathrm{N}_{2}$ stream.

The magnetization from the bare substrate was measured before the formation of the SAMs at different temperatures (Fig. 11(a)). At low temperatures, the substrates have a paramagnetic behavior that turns to diamagnetic at $150 \mathrm{~K}$. This behavior is typical of the paramagnetic impurities. The same results were found 


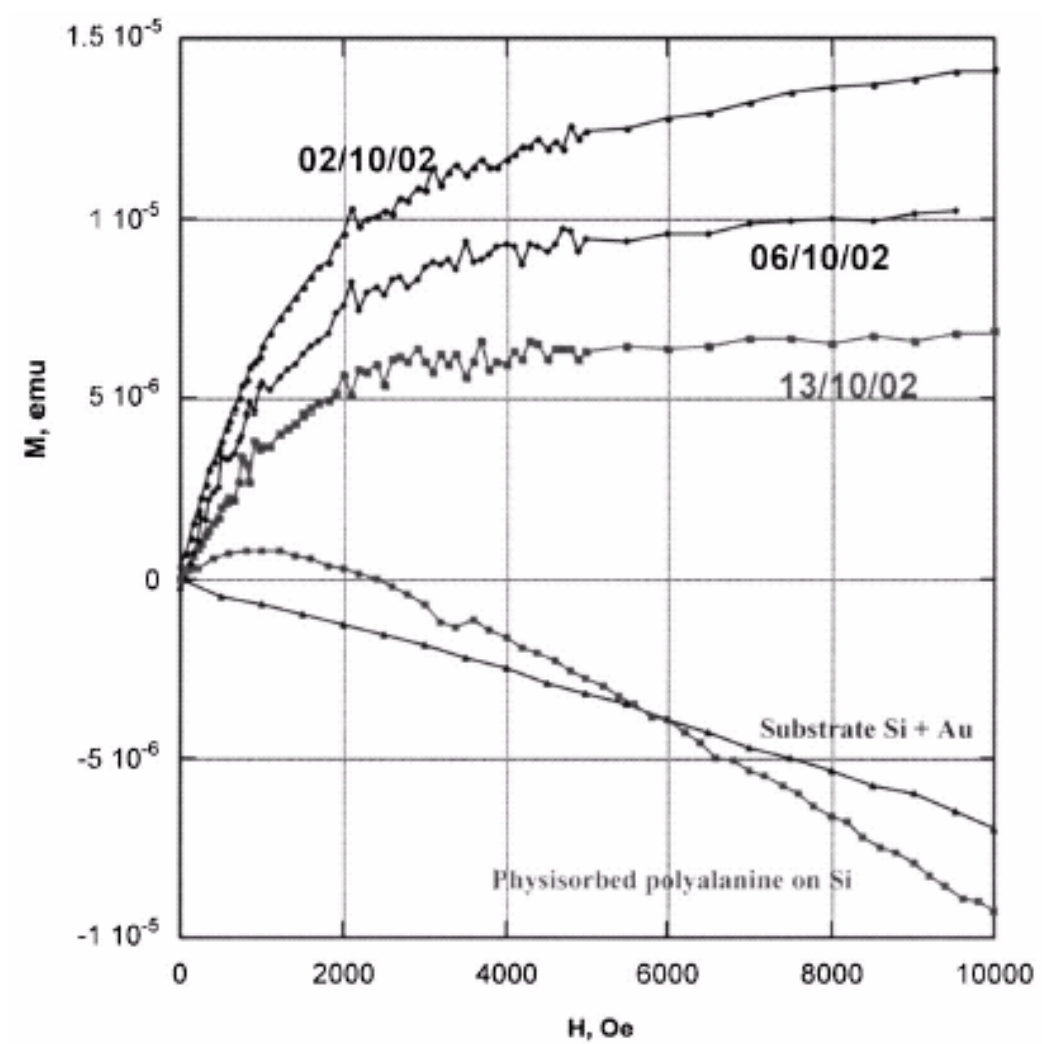

Fig. 10. Room temperature magnetization versus field for a polyalanine monolayer chemisorbed on the gold film. The different curves were measured at different dates (from Ref. 19). Copyright (2003) by the American Institute of Physics. Reproduced with permission of the authors.

when the field is applied parallel and perpendicular to the substrate surface, as expected from dispersed magnetic impurities. Figure 11(b) presents the results before and after the chemisorption of the Lewis molecules, applying the field parallel and perpendicular to the surface at $5 \mathrm{~K}$. The magnetization curves are similar for the substrate and the coated surface when the field is parallel to the sample. However, when the field is perpendicular, a ferromagnetic component arises.

Figures 11(c) and 11(d) show the magnetization curves after removing the signal of the substrate, with the field applied perpendicular (Fig. 11(c)) and parallel (Fig. 11(d)) to the Au surface.

The most striking results are:

(i) The appearance a ferromagnetic-like behavior in the capped Au surface despite the paramagnetic or diamagnetic behavior of the bare substrate.

(ii) The magnetism is only observed when the field is applied perpendicular to the surface.

(iii) The giant values that reach the magnetization, about $80 \mu_{B}$ per surface atom. 


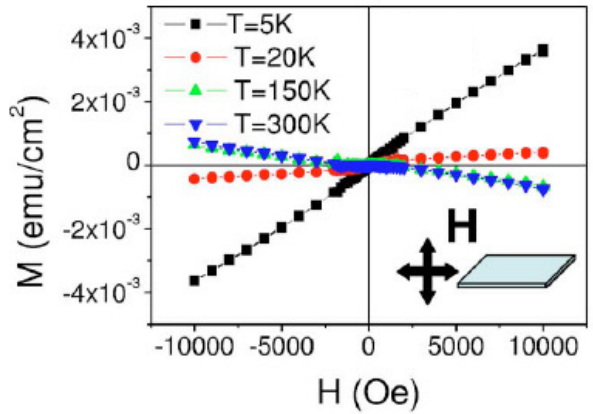

(a)

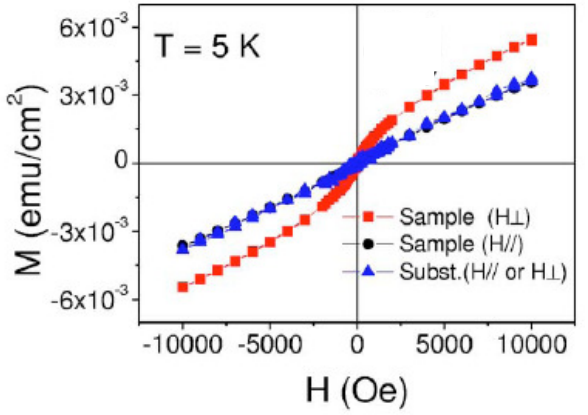

(b)

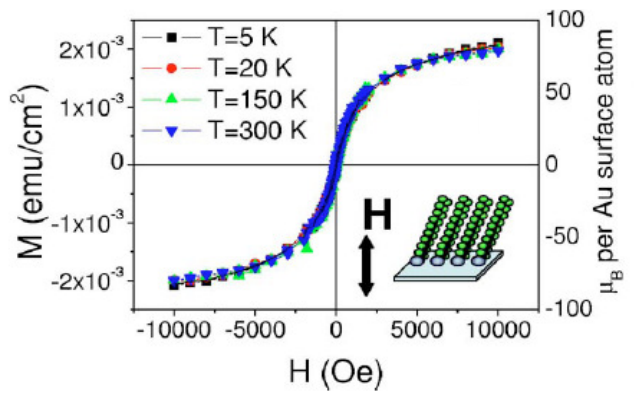

(c)

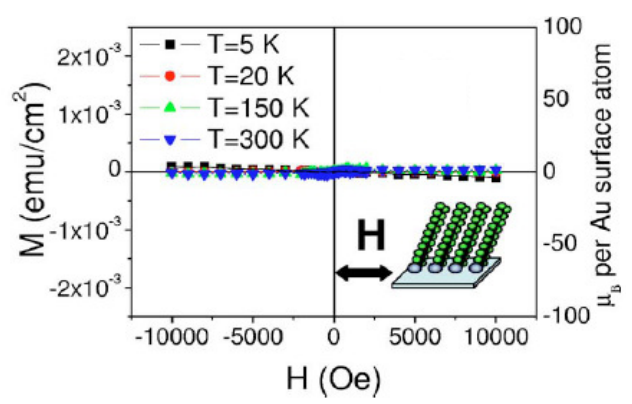

(d)

Fig. 11. Magnetization curves for (a) the bare substrate with $H$ perpendicular to the surface; the same result was found for $H$ parallel, (b) the bare substrate and the sample with $H$ parallel and perpendicular at $5 \mathrm{~K}$. (c) Difference between the magnetization curves for the substrate and the sample with $H$ perpendicular to the surface and (d) $H$ parallel to the surface. Adapted from Ref. 29.

(iv) The magnetization is independent of the temperature. This is the fingerprint of giant magnetic anisotropy that blocks the magnetic moments so that thermal energy cannot reorient them even at room temperature.

As for the case of NPs, surface plasmon absorption experiments carried out on thiolated gold thin films can help to elucidate the origin of their ferromagnetic-like behavior. The characteristics of a surface plasmon-polariton strongly depend on the chemical species adsorbed on the metal-air interface. An increasing number of chemical biosensors are currently being developed based on this dependency. ${ }^{23}$

We have performed Surface Plasmon Resonance (SPR) experiments in the Kretschmann-Raether configuration, ${ }^{24}$ both with clean and thiolated thin films. The thin films were grown by thermal evaporation in vacuum on borosilicate substrates. To ensure a higher flatness, the samples are further annealed in a flame. The slides were placed on a prism both optically coupled with the adequate indexmatching liquid. The angular dependence of the reflectivity using a $p$-polarized $\mathrm{He}-\mathrm{Ne}$ laser was recorded. Figure 12(a) shows the measured reflectivities in a Au 
film before and after thiols deposition. The excitation of the surface plasmons by the light produces its absorption and the sharp decreasing of the reflectivity. It is clearly observed that the presence of the thiols shifts the maximum toward higher values and the band becomes broader. Theoretical models based on the Fresnel reflectivity equations for a $N$-layer system actually predict that if the effect of thiols is merely to introduce a layer with different refraction index in the top of the $\mathrm{Au}$ film, the surface plasmon resonance band should shift in the observed direction, but with a smaller magnitude and without increase in the width. ${ }^{25}$ Thus, it is inferred that the thiol layer is not just an inert layer but interacts with the Au film modifying in some way its electronic properties.

In order to analyze this hypothesis, we have measured the dependence of the surface plasmon on the gold film thickness. The results (Fig. 12(b)) clearly show that for a layer thinner than the optimal one, the plasmon resonance peak shifts to higher values and its FWHM broadens. These observations suggest that the electronic affinity of sulfur in the capping molecules, as in the case of the NPs, could be partially blocking the free plasma oscillation of the gold layer close to the interface, thus reducing the effective thickness of the film as it happens in the case of the nanoparticles described above.

Vager and Naaman proposed the first explanation for the giant magnetic moments of thin films. ${ }^{26}$ They hold that after the organic molecules absorption, electrons flow from the Au to the organic layers and form bosons with large orbits that stand a large magnetic moment. We showed that in their explanation they missed a diamagnetic term in the Hamiltonian. ${ }^{27}$ An alternative explanation has been recently proposed..$^{28,29}$ The SAMS formed onto the gold surfaces spontaneously form domains. ${ }^{30}$ The charge transfer at the $\mathrm{Au}-\mathrm{S}$ bond induces a contact potential. ${ }^{31}$ We consider that the capped region is circular with a radius $\xi$. For this circular

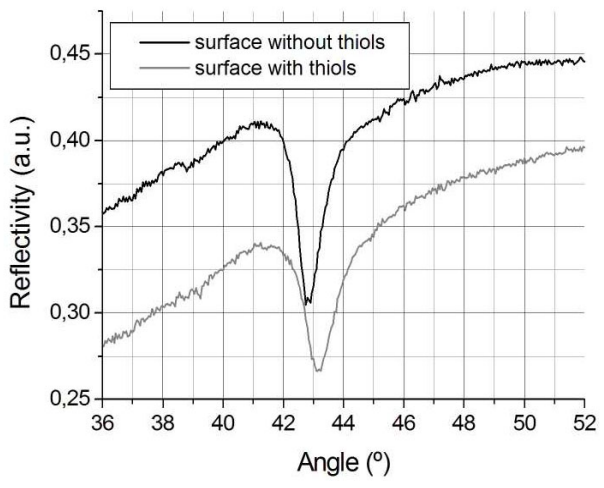

(a)

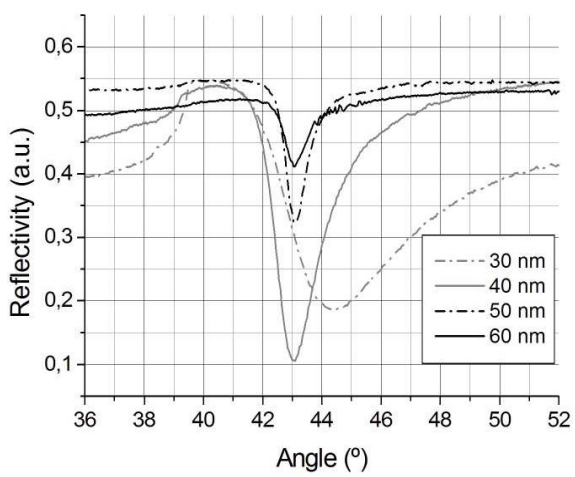

(b)

Fig. 12. Reflectance measurements of the surface plasmon resonance (a) on a clean gold film (higher curve) which was later thiolated (lower curve). A shift in the extinction angle and a broadening in the peak is observed (b) on clean gold films for different thicknesses, ranging from 30 to $60 \mathrm{~nm}$. 


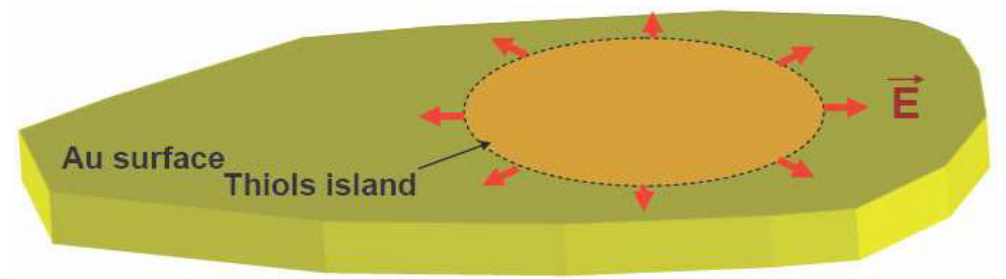

Fig. 13. Schema of a gold surface with a thiol island and the direction of the electric field that arises.

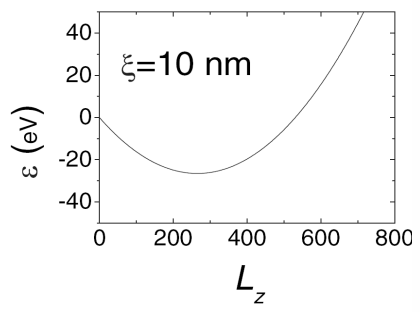

(a)

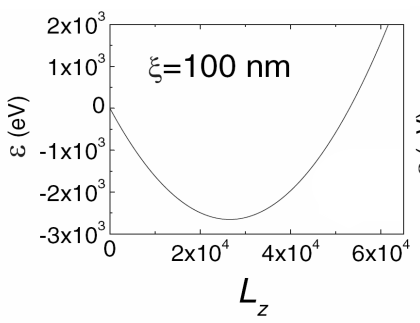

(b)

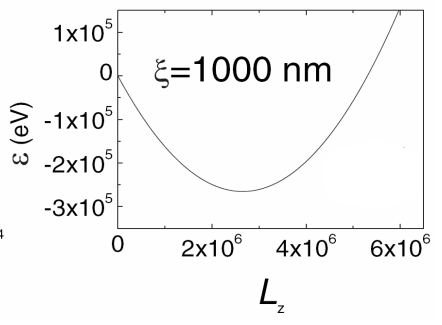

(c)

Fig. 14. Orbital energy $\varepsilon$ of the electron in the localized orbit as a function of $L_{z}$ for different radius. Adapted from Ref. 28.

island of radius $\xi$, a radial electric field appears at the border as it is illustrated in Fig. 13.

This potential step traps quasifree electrons in atomiclike orbits of radius $\xi$. The Hamiltonian for this electron, including the spin orbit coupling is

$$
H=\frac{L_{z}^{2} \hbar^{2}}{2 m \xi^{2}}-\alpha_{r} L_{z} s_{z} h^{2} .
$$

The first term of this expression corresponds to the angular moment while the second corresponds to spin orbit coupling. With this $H$, the mimimum energy corresponds to:

$$
L_{z}=m \xi^{2} \alpha_{r} s_{z},
$$

where $\alpha_{r}$, which is the order of the splitting strength, is dependent on the spin-orbit interaction. The value, according to LaShell et al. ${ }^{32}$ is $\alpha_{r} h^{2}=0.4 \mathrm{eV}$ for $\mathrm{Au}(111)$ surfaces.

The value that minimizes the orbital energy, $\varepsilon$, of the electron in this localized orbit is dependent on the spin orbit coupling, the orbit radius $\xi$ and on the orbital momentum $L_{z}$. Figure 14 shows the orbital energy $\varepsilon$ dependence on the quantum number $L_{z}$ for three different values of $\xi$. If the island is large enough, the energy could reach the minimum value when the orbital momentum has giant values.

The total angular momentum per surface atom trapped in the orbit with spin up and spin down is similar. The orbit states with $L_{z} \uparrow$ and $L_{z} \downarrow$ are equally populated 


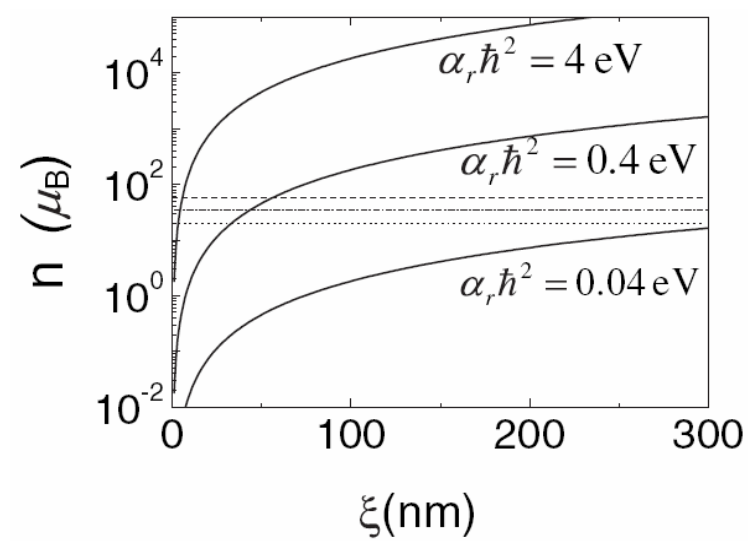

Fig. 15. Magnetic moment, in number of Bohr magnetons per surface atom $[\mathrm{Au}(111)]$ as a function of the orbit radius for different values of $\alpha_{r}$. Experimental results reported and measured are also plotted as horizontal lines: (dashed line) fresh samples; (dashed-dotted line) aged 5 days; (dotted line) aged 10 days. Adapted from Ref. 28.

vanishing the total $L_{z}$. For each electron, spin and orbital moments are coupled. Exchange interactions between electrons tend to align their spins. The spin-orbit coupling ( $\sim 0.4 \mathrm{eV}$ at Au surface) leads to $L_{z}$ alignment and, as a result, $L_{z}$ does not vanish. Therefore, a permanent magnetic moment with spin and giant orbital momenta arises at the surface.

Assuming that the electrons are trapped and spin-polarized, the number of Bohr magnetons per surface atom linked to an organic molecule and associated with the orbital angular momentum is given by $n\left(\mu_{B}\right)=m^{2} \xi^{2} \alpha_{r} a^{2}$, where $a$ is an effective lattice constant. Figure 15 shows $n\left(\mu_{B}\right)$ as a function of the island radius and for different values of $\alpha_{r}$.

The values for $n\left(\mu_{B}\right)$ explain some of the most relevant aspect of this surprising magnetic behavior:

(i) The magnetic moments come from orbital momentum, which implies high magnetic anisotropy.

(ii) The quasifree electrons are trapped in localized states, which produces a reduction in electron mobility as observed in plasmon measurements.

(iii) The magnetic moments can reach giant values if $\xi$ is large.

(iv) The origin of orbital moment alignment lies in exchange interactions between spins.

(v) Degradation of organic layers produces a degradation of magnetic properties, due to the decrease in size of the islands.

(vi) Since the $z$ axis should be very well defined through the domain, very flat surfaces are needed to have regions with large $\xi$.

(vii) For NPs, as the $z$ axis is not well defined, $\xi$ is small. Hence, the magnetic moment per atom is three orders of magnitude smaller than for thin films. 


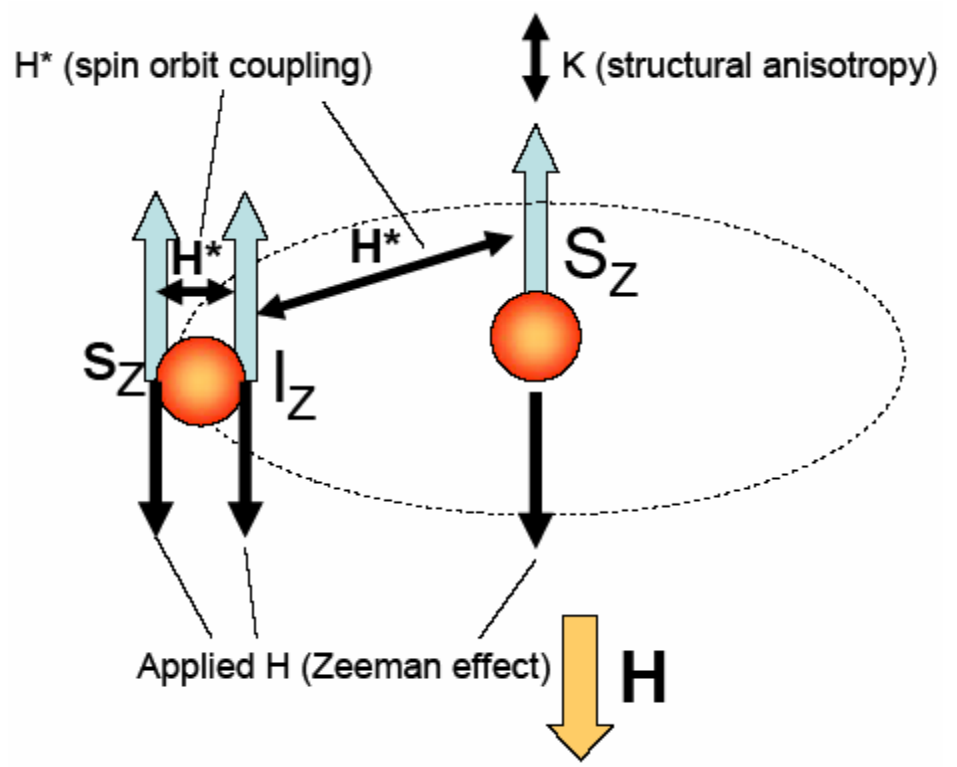

Fig. 16. Scheme of the different magnetic moments and the interaction controlling their orientation. The relative orientation of $S_{Z}, s_{Z}$ and $l_{Z}$ is fixed by $H^{*}$. The structural anisotropy acts only on $S_{Z}$ while the reversal magnetic applied field acts on all of them. Adapted from Ref. 29.

The origin of the giant magnetic anisotropy is also related with the origin of the magnetic moments. ${ }^{29}$ If the surface has a localized spin, $S_{z}$, the conduction electrons will rotate around it due to the spin-orbit coupling. These electrons have an orbital momentum $l_{z}$ and a spin component $s_{z}$.

The spin-orbit interaction per atom couples the localized charge and spin to the spin and orbital momenta. So the contributions to the magnetic moments at the surface come from (Fig. 16):

(a) Localized magnetic moment, $g \mu_{B} J=g \mu_{B}(L+S)$, where $g$ is the Landé factor and $J$ the total angular momentum of a localized electron.

(b) $\mu_{B} l_{z}$ is the orbital magnetic moment per atom induced on conduction electrons.

(c) $2 \mu_{B} s_{z}$ that is the magnetic moment per atom associated with the spin of the conduction electrons with orbital momentum $l_{z}$.

The conduction electrons rotate around localized charges and/or localized spins along orbits contained on the surface and, consequently, $l$ can only have $z$ component. Therefore, rotation of orbital magnetic moment is meaningless since it would mean that the electrons leave the metallic surface. However, for any given $l_{z}$, its reversal does not change the energy provided a simultaneous reversal of $\left(s_{z}+S_{z}\right)$.

For NPs, the value orbital magnetic moment is of the same order than the spin magnetic moments and both have contribution to the macroscopic magnetization. 
However, in films the orbital moment contribution is giant and its contribution is the only one observed.

The localized spins even though subjected to possible anisotropies independent of the orbital motion, are mainly blocked by the effective spin-orbit field, $H^{*}$, given by:

$$
\mu_{0} H^{*}=\alpha_{r} h^{2} l_{z} / \mu_{B}
$$

This field strength $\mu_{0} H^{*}$ is close to $1000 \mathrm{~T}$ for $\alpha_{r} \hbar^{2}=0.4 \mathrm{eV}$, that is the case of spins localized on gold surfaces with $l_{z}=1$. Furthermore, for Au films the localized spins are blocked through an effective field $10^{2}$ times higher due to the giant value of $l_{z}$ that can approximate $10^{2}$. Thus, for the case of gold in both forms, films and NPs, the enormous strength of $H^{*}\left(10^{5}\right.$ and $10^{3} \mathrm{~T}$, respectively) indicates that $S_{z}$, $s_{z}$ and $l_{z}$ remain stiffly coupled under any applied field. This giant effective field cannot induce any reversal of the magnetic moments since its direction reverses with them, but accounts, however, for the blocking of moments observed in $\mathrm{Au}$ NPs up to room temperature.

The lack of hysteresis observed in gold films indicates that at zero applied field, the number of orbits with quantum number $l_{z}$ is equal to that with $-l_{z}$ and that transitions between them do not need to overcome any barrier. Consequently, a very weak magnetic field enables $l_{z}$ reversal.

The hysteresis observed for Au NPs can be explained as follows. The localized spins are also subjected to the local structural anisotropy with constant $k$ per atom and with an easy axis that we assume perpendicular to the surface. Therefore, if the localized spin were isolated, they will reverse for an opposite field of strength $H=2 k / \mu_{0} \mu_{B}$. However, the applied field acting on $S$ and trying to reverse it also acts on the orbital moment that is rigidly linked to $S$, as illustrated by Fig. 16 . Consequently, the reversal will take place at a field

$$
H_{K}=\frac{2 k}{\mu_{0} \mu_{B}\left(2 s_{z}+2 S_{z}+l_{z}\right)} .
$$

For thin films, $l_{z}$ is in the order of 100 , the reversal field is negligible, the curve does not present hysteresis, and only demagnetizing effects are observed. For NPs, as $l_{z}$ is of the order of unity, the reversal is fairly larger than for thin films (for which $l_{z}$ is larger) so some hysteresis may be still be observed.

The magnetic moments are blocked by $H^{*}$, rather than by $k$, and can remain blocked up to above $300 \mathrm{~K}$ when the NPs size is even smaller than $1 \mathrm{~nm}$. Consequently, the magnetization under constant applied field does not depend on temperature over a broad interval between 5 and $300 \mathrm{~K}$.

In summary, we have shown our recent experiments for the study of the surprising ferromagnetic-like behavior that arises at the Au surfaces, in NPs and thin films. This magnetic behavior is induced by the $\mathrm{Au}-\mathrm{S}$ bonds. Although the magnetic properties of the two systems are not the same, due to the different characteristics of NPs and thin films, the origin of the magnetism is the same. It is a magnetism 
independent of the temperature, which shows high anisotropy. In the case of thin films the magnetic moments may reach giant values. We proposed that the orbits of conduction electrons at the Au surface are responsible for the magnetic moments. These orbits are determined by the size of thiol packs, and for NPs are limited to atomic distances, because of the lack of planar surfaces. The large anisotropy is due to the huge spin-orbit coupling of Au surfaces.

\section{References}

1. S. Chikazumi, Physics of Ferromagnetism, 2nd edn. (Clarendon, Oxford, 1997).

2. P. Esquinazi et al., Phys. Rev. Lett. 91 (2003) 227201.

3. M. Venkatesan, C. B. Fitzgerald and J. M. D. Coey, Nature 430 (2004) 630.

4. P. Gambardella et al., Science 300 (2003) 1130.

5. P. Crespo et al., Phys. Rev. Lett. 93 (2004) 087204.

6. Yamamoto et al., Phys. Rev. Lett. 93 (2004) 116801.

7. D. A. Papaconstantopoulous, Handbook of Band Structure of Elemental Solids (Plenum Press, New York, 1986).

8. E. Vogt, in Magnetism and Metallurgy I, ed. A. E. Berkowitz and E. Kneller (Academic Press, New York, 1969), p. 252.

9. P. Zhang and T. K. Sham, Appl. Phys. Lett. 81 (2002) 736.

10. P. Zhang and T. K. Sham, Phys. Rev. Lett. 90 (2003) 245502.

11. M. Brust, M. Walker, D. Bethell, D. Schiffrin and R. Whyman, J. Chem. Soc. Chem. Commun. (1994) 801.

12. M. J. Hostetler et al., Langmuir 14 (1998) 17.

13. I. Carabias et al., E-Nano Newsletters 4 (2006) 11.

14. U. Kreibig and M. Völlmer, Optical Properties of Metal Clusters (Springer-Verlag, Berlin, 1995).

15. H. Hövel, S. Fritz, A. Hilger, U. Kreibig and M. Völlmer, Phys. Rev. B 48 (1993) 18178.

16. J. A. Creighton and D. G. Eadon, J. Chem. Soc. Faraday Trans. 87 (1991) 3881.

17. G. Mie, Ann. Phys. 25 (1908) 377.

18. M. A. Garcia et al., Phys. Rev. B 72 (2005) 241403(R).

19. Carmeli et al., J. Chem. Phys. 18 (2003) 10372.

20. Hegner et al., Surf. Sci. 291 (1993) 39.

21. C. Tromas et al., Langmuir 21 (2005) 6142.

22. J. M. de la Fuente and S. Penades, Tetrahedron: Asymmetry 13 (2002) 1879.

23. Surface Plasmon Resonance Based Sensors, ed. J. Homola, Springer Series on Chemical Sensors and Biosensors, Vol. 4 (Springer, 2006).

24. H. Raether, Surface Plasmons (Springer-Verlag, Berlin, 1988).

25. M. Yamamoto, Review of Polarography 48 (2002) 209.

26. Z. Vager et al., Phys. Rev. Lett. 92 (2004) 087205.

27. A. Hernando and M. A. Garcia, Phys. Rev. Lett. 96 (2006) 029703.

28. A. Hernando, P. Crespo and M. A. Garcia, Phys. Rev. Lett. 96 (2006) 057206.

29. A. Hernando et al., Phys. Rev. B $\mathbf{7 4}$ (2006) 052403.

30. E. Barrena, C. Ocal and M. Salmeron, J. Chem. Phys. 111 (1999) 9797.

31. T. Ichii et al., Nanotech. 15 (2004) S30.

32. LaShell et al., Phys. Rev. Lett. 77 (1996) 2419. 